Eröffnungsbeitrag

Hartmut Böhme*

\title{
Perspektiven der Kulturwissenschaft in historischer und gegenwartsanalytischer Perspektive
}


Abstract: After observations on Studies of Culture (Kulturwissenschaften) in relation to their theoretical foundation and their subjects in the last decades, the functioning (style in the sense of Ludwik Fleck) of ,Kulturwissenschaft' will be examined not systematically but exemplarily in four fields: (i) the functions of the so-called liberal and applied arts in the last 250 years, (ii) constellations of subjectivity in industrial society in the nineteenth century, (iii) the relation between security and risk in the present time and (iv) the relation of culture and religion in the polycentric, post-religious, but also post-Enlightenment world. Considerations on the prospects of a historic ,Kulturwissenschaft' in relation to Anglo-American trends follow this course.

Keywords: Fachentwicklung der Kulturwissenschaft, Kulturgeschichte, Rousseau, Arbeitsgesellschaft, Erschöpfung, Moderne, Religiosität, Perspektiven

*Prof. Dr. Hartmut Böhme: Institut für Kulturwissenschaft, Humboldt-Universität Berlin, Unter den Linden 6, D-10099 Berlin, email: HHBoehme@gmx.de

\section{Kulturwissenschaft als ,Fach'?1}

Lassen Sie mich mit einem Zitat von Max Scheler von 1929 beginnen: „Wir sind in der ungefähr zehntausendjährigen Geschichte das erste Zeitalter, in dem sich der Mensch völlig und restlos problematisch geworden ist: in dem er nicht mehr weiß, was er ist; zugleich aber auch weiß, dass er es nicht weiß." (Scheler 1955: 120) Scheler spricht hier vom Menschen im Kollektiv-Singular; das tun wir Kulturwissenschaftler nicht: für uns gibt es nur Menschen als Individuen; und es gibt nicht die Kultur, sondern nur Kulturen. Aber es gibt durchaus strukturelle Merkmale und fundierende Mechanismen, durch welche sich Kulturen von dem, was nicht Kultur ist, und sich Menschen

1 Der folgende Text behält den Wortlaut des Vortrags bei der Gründungsversammlung der Kulturwissenschaftlichen Gesellschaft in Koblenz im Januar 2015 bei und ergänzt diesen lediglich um Anmerkungen. Inhalte und Stil der Kulturwissenschaft sind nicht aus einer Theorie der Kultur, die unser Fach allerdings auch benötigt, schlicht abzuleiten. Darum werden nur exemplarische Felder historischer Forschung vorgestellt, die eingerahmt sind von Überlegungen zum gegenwärtigen und vielleicht künftigen Satus des Faches Kulturwissenschaft. Vollständigkeit der Gegenstände, ein Überblick über Methoden und Theorien und ihre Geschichte, also eine Systematik des Faches sind hier, im Rahmen eines Vortrags, nicht angestrebt. von Nicht-Menschen unterscheiden. Scheler operiert ferner in der longue durée; das tun wir auch, doch ebenso untersuchen wir engbegrenzte Objektfelder in chronotopischer Verdichtung. Der Philosoph, ganz Zeitgenosse der Moderne, geht von einer epochalen Identitätsdiffusion aus, die mit Reflexivitätssteigerung verbunden ist: das ist das Zeitbewusstsein des Romans Der Mann ohne Eigenschaften von Robert Musil. Beide, Philosophie und Literatur, diagnostizieren für die ausdifferenzierte Moderne den Verlust substantieller Werte und verbindlicher Lebensweisen, d.h. eine exponentielle Zunahme von Kontingenz. Doch diese Selbst-Auflösung ist selbst ein Phänomen der für die Moderne typischen Reflexivitätszunahme, oder, wie Niklas Luhmann sagt, der Beobachtungsbeobachtung. Diese reflexive, zweite Stufe von Beobachtung ist ein Konstituens der Kulturwissenschaft. Wir beobachten, analysieren, interpretieren und erklären historisch vorfindliche Beobachtungen; und diese sind nichts anderes als kulturelle Konfigurationen und Prozesse.

Das klingt so, als wäre die Kulturwissenschaft das Zentralorgan der Philosophischen Fakultät. Davon kann keine Rede sein. Erwartungsgemäß haben sich die geisteswissenschaftlichen Disziplinen nicht einfach zu Kulturwissenschaften gewandelt - das wäre illusorisch; aber es wäre 
auch nicht wünschenswert. Eine kulturwissenschaftliche Modernisierung ist nur sinnvoll im komplementären, ergänzenden oder erweiternden Verhältnis zu den Traditionen der übrigen geisteswissenschaftlichen Fächer. Andererseits hat sich vieles geändert: Anträge auf große Drittmittelprojekte (Sonderforschungsbereiche u.ä.) haben umso eher eine Erfolgsaussicht, als sie sich kulturwissenschaftlich u.d.h. zumeist: interdisziplinär ausweisen. Ganze Fächer haben inr Profil grundlegend reformiert (etwa von der Volkskunde zur Europäischen Ethnologie, vgl. Zimmermann 2007). Viele Philologien, etwa die Germanistik, aber auch die Theaterwissenschaft, haben sich im Kern kulturwissenschaftlich renoviert. Auch in Leitfächern der alten Fakultäten wie der Philosophie, Geschichte, Kunstgeschichte und Soziologie gibt es Diskussionen um die kulturwissenschaftliche Wende. In jüngeren Fächern, deren Gründung nicht lange zurückliegt, wie z.B. in der Kommunikations- und Medienwissenschaft, nehmen kulturwissenschaftliche Fragestellungen ein breiten Raum ein.

Mit der Kulturwissenschaft im Singular sieht es jedoch anders aus: Nur an einigen Universitäten haben sich Disziplinen mit dem Titel Kulturwissenschaft gebildet - allerdings mit ganz verschiedenen Ausrichtungen. Grosso modo gilt: Kulturwissenschaft im Singular will man nicht, weder in der DFG noch an den Universitäten. Kulturwissenschaft soll kein Fach, sondern eine Orientierung für die Fächer der Philosophischen Fakultät sein. Das kann geändert werden: Denn zwischen der Institutionalisierung des Fachs Kulturwissenschaft und der kulturwissenschaftlichen Perspektivierung vieler Fächer der Philosophischen Fakultäten besteht kein Widerspruch. ${ }^{2}$

Neben der Kulturwissenschaft gibt es auch andere Felder, die keine ,Fächer' sind, aber dennoch Querschnittsfunktionen übernehmen. Ich erwähne nur die Semiotik, die als Kultursemiotik zur Rekonstruktion der Zeichen- und Bedeutungsprozesse von Kulturen basal sind. Ich denke an die Kulturökologie (Finke 2008; Zapf 2008) ein klarer Fall für eine praktische Kulturwissenschaft. Ich erinnere an die Kulturtechniken von Bild, Schrift und Zahl, durch welche neue 'Felder'

2 Assmann (2006); Därmann/Jamme (2007); Nünning (2005); Liebsch/Rüsen/Straub (2004); Kittsteiner (2004); Benthien/Velten (2002); Böhme/Matussek/Müller (2007). ausdifferenziert wurden, die interdisziplinäre Vernetzungen herzustellen, aber auch die natur- und technikwissenschaftlichen Praxen zu integrieren vermögen (Krämer/Bredekamp 2003). Uns besonders nahe stehen die Medienwissenschaften, die in den tradierten Fächern ein Bewusstsein dafür geweckt haben, dass kulturelle Praktiken überwiegend medial konstituiert und prozessiert werden - nicht nur, seit es technische Medien gibt, sondern auch dort, wo die sog. natürlichen Medien (z.B. die Stimme, Luft) die Vermittler kultureller Handlungen, Kommunikationen und Zirkulationen darstellen. Kurzum: Medienwissenschaften sind Medienkulturwissenschaften.

Im Allgemeinen ist das Bild von Kultur in den Kulturwissenschaften zu sehr auf symbolische Kommunikation und soziales Handeln zentriert: Dabei bleibt außer Blick, dass menschliche Kulturen nur analysiert werden können in den historischen Verhältnissen zu dem, was nicht-menschlich ist: das sind die Dinge, die Tiere und Pflanzen, die Regionen und Landschaften, das Wetter und das Klima. Wir vergessen zu leicht, dass es im Blick auf die artifiziellen Dinge, die ,kultivierten' Tiere und Pflanzen oder die Kulturgeographien eine weltweite kulturgeschichtliche Forschung gibt, zu der wir einen lebendigen Kontakt brauchen, wenn man die programmatische Absicht auf ,Kulturwissenschaft' einlösen will.

Aus diesen Beobachtungen haben wir gelernt: Kulturwissenschaft wird sich nicht durchsetzen, wenn man sie nur als ein Additionsspiel von aktuellen Erweiterungen etablierter Disziplinen betreibt. Es bedarf stärkerer theoretischer Anstrengungen und vor allem einer von Kulturtheorie(n) angeleiteten Bestimmung der ,Felder' kulturanalytischer Arbeit. Diese entstehen gerade nicht durch Summation der Vorschläge aus den Sichten der Einzeldisziplinen.

Im Folgenden skizziere ich, bezogen auf Entwicklungen der Moderne in den letzten 250 Jahren (der historische Horizont der Kulturgeschichte ist natürlich viel weiter), (1) die Funktionen der sog. freien und der nützlichen Künste, die beide zu den Gegenständen unserer Fächer gehören, (2) Konstellationen der Subjektivität in der Industriegesellschaft des 19. Jahrhunderts, (3) das Verhältnis von Sicherheit und Risiko heute sowie (4) die Beziehung von Kultur und Religion in der polyzentrischen, postreligiösen, aber auch postaufklärerischen Welt. Große Themen auf engstem Raum! 
Doch will ich nur beispielhaft zeigen, worin sich ein kulturwissenschaftlicher Blick auszeichnet und dass inm Phänomene in den Fokus geraten, die es berechtigt erscheinen lassen, von einem spezifisch kulturwissenschaftlichen Umgang mit historischen Objekten und Feldern zu sprechen.

\section{Das Nutzlose und das Nützliche}

1762 war einer der radikalsten Vertreter der Aufklärung mal wieder auf der Flucht. Von Paris aus hatte er sich ins schweizerische Städtchen Môtiers zurückgezogen, das zum Herrschaftsgebiet des preußischen Königs gehörte. Hier war der berüchtigte Exzentriker dem calvinistischen Pfarrer und den Bauern unliebsam geworden, so dass sie ihm mit Steinen die Fenster einwarfen. Bereits in Paris, dann auch in Genf hatte man seine Schriften verbrannt - und einen Haftbefehl ausgestellt. So musste man nicht paranoid sein, um sofort nach der "Steinigung" (lapidation, wie er schreibt) erneut zu fliehen: auf die Isle St. Pierre im Lac de Bienne im Jura - procul negotiis und das heißt hier: fern des "Tumultuarischen des gesellschaftlichen Lebens" und einer "vollständigen Weltabwendung" (Rousseau 2012: 139; 85). Über diesen idyllischen Aufenthalt schreibt JeanJacques Rousseau zwölf Jahre später die berühmten Träumereien eines einsam Schweifenden. Warum erzähle ich das?

In der „fünften Träumerei" beschreibt Rousseau folgende Situation: Er lässt sich mit einem Boot über den See treiben und versinkt in stundenlange Träumereien, "wirr zwar, aber herrlich", „ohne genau umrissenen oder fest gefügten Gegenstand". Ähnliche Situationen "jeglicher Mühsal des Denkens enthoben", wodurch er indes umso mehr sein "Dasein voll Freude [zu] spüren" vermag, erlebt er auf der Insel oft. Die Zeit wird aufgehoben, jede Minute ist wie die andere, jeder Tag wie der vorige. Es sei ein "dauerhafter Zustand" ',"wo Zeit nichts mehr gilt, wo stets Gegenwart herrscht, ohne jeden Merkstein solcher Dauer und ohne jede Spur einer Abfolge, aber auch ohne jedes Gefühl von Mangel oder Wonne, von Freud oder Leid, von Wunsch oder Angst, außer um unser Dasein selbst, das ganz von diesem Gefühl ausgefüllt wird" (Rousseau 2012: 131, 133, 135). Es ist ein Zustand der See- lenruhe (der epikureischen aтараछia), jenseits jeder moralischen Verpflichtung, jeder Arbeitsdisziplin und jeder Wahrheitsanstrengung. Rousseau bildet in sich selbst die Insel, auf der er lebt. Und diese Insularität, fern jeder Robinsonade, in der es stets um Selbsterhaltung und Arbeitsethos geht, ist der Inbegriff der Ästhetik. Das Ästhetische teilt sich hier gerade nicht als werkschaffende Kreativität, sondern als selbstgenügsamer, nutzloser, aber erfüllter Augenblick mit. Kurz, es ist das Glück, - die Erfahrung des verweilenden Augenblicks, den Faust, der Weltengierige, stets sucht und nie erreicht. Faust, der rastlos Schaffende, ist der Gegentyp zu Rousseau im Boot, dem Genießenden.

Rousseau ist nicht nur Philosoph, sondern auch Künstler und Musiker, der die Hetze und Unruhe der Zeit nur zu genau kennt. Hier auf St. Pierre lernt er eine neue Art des Ästhetischen kennen: das Gefühl vollkommener Immanenz des Daseins, worin "man sich selbst genügt" „wie Gott". Das nennt Peter Sloterdijk den „Urknall der modernen Subjektivität" (Sloterdijk 2003: 23). Indes, es ist keine Explosion, sondern "ein fast unmerkliches Geschehen von eher implosivem oder kontemplativem Charakter" (Rousseau 2012: 136). ${ }^{3}$ Es ist schon deswegen kein Urknall, weil diese Form von Moderne deutlich auf alteuropäische Topoi des anti-urbanen Landlebens zurückgeht (Sengle 1963; Frühsorge 1993).

Von hier aber gehen die Theorien ästhetischer Autonomie aus, die Ansprüche der Kunst auf Nutzlosigkeit und Amoralität, auf Genuss und reine Gegenwart - von Schiller bis zu Adornos ästhetischer Theorie, die ihren Impuls aus der Figur der Negativität bezieht: In der Kunst sei aller gesellschaftlicher Anspruch gelöscht und eben das mache ihren unverzichtbaren Wert aus. Denken wir an Joris-Karl Huysmans, Oscar Wilde oder Hugo von Hofmannsthal. Denken wir an die Selbstgenügsamkeit von Farbe und Form, die absolute Malerei von Cezanne bis zu Barnett Newman.

Rousseaus Ästhetik der Präsenz (vgl. dazu Mersch 2002) ist jedoch eine Geburt der Aufklärung nicht anders als die Erfindung der nützlichen Künste. ${ }^{4}$ Die Encyclopédie von d'Alembert und

3 Vgl. zu diesem Zusammenhang Sloterdijk 2001: 25-28. 4 Nützliche Künste bezeichnen schon vor der Aufklärung vor allem technische Fertigkeiten, Verfahren, Apparate 
Diderot hatte programmatisch die Kulturtechniken, Gewerke und Praktiken in den Vordergrund gerückt. Die Künste sollten ihre gesellschaftliche Nützlichkeit darstellen. Der Widerspruch zwischen den sog. freien und den nützlichen Künsten zieht sich durch die Geschichte besonders der Kunsthochschulen. Doch gerade die Kulturwissenschaft hat hinsichtlich der artes keinerlei Probleme damit, beide Seiten, die praktisch-funktionale und die ästhetisch-autonome gleichermaßen zu berücksichtigen.

Vergessen wir nicht, dass Rousseau keineswegs nur im Boot lag und träumte: vita contemplativa, sondern das Eiland im Bieler See kartographierte, um die Pflanzen systematisch aufzunehmen; dass er die unbewohnte Nachbarinsel mit Kaninchen besiedelte und sich mit dem Verweser des kleinen Landguts über Agrikultur beriet: vita activa (Arendt 1981). Und selbstverständlich schrieb er, der auf der Insel sein Schreibzeug nicht anzurühren vorgab, später ein ganzes Buch über den Aufenthalt auf St. Pierre: reflexive Kultur.

Vergessen wir nicht, dass europaweit Gesellschaften gegründet wurden nach dem Vorbild der Royal Society for the Encouragement of Arts, Manufactures and Commerce. Die Autonomie der Kunst war ein Theorie-Phantasma des Idealismus, der sich über seine materiellen und sozialen Bedingungen hinwegtäuschte. Die glückliche Nutzlosigkeit der Kunst ist eine abstrakte Grenzbestimmung, die bis heute kaum etwas mit der Realität der Kunstpraxis zu tun hat. Sie ist dennoch wichtig, um die Kunst vor politischer Zensur, moralischen Zwecksetzungen und szientifischer Funktionalisierung zu verteidigen. Dem gegenüber standen die nützlichen Künste. An deren Entwicklung meldete niemand ein so lebhaftes Interesse an wie das Wirtschaftsbürgertum, das aus demselben Stamm wuchs wie die Kunstautonomie, nämlich der Aufklärung. Die Regime der Arbeit und der Industrie gaben den Rahmen für Berufsbilder ebenso her wie für die Gestaltungsprinzipien jener Artefakte, die nur als Waren eine Chance für ihren Auftritt in der Welt hatten. Zwischen kapitalistischen Verwertungsimperativen

und Maschinen, also solche technomorphen Prozesse, die zunächst an den (Kunst- und) Gewerbeschulen, später dann an den technischen Universitäten gelehrt und gelernt wurden. und der Selbstbehauptung der Kunst, die frei und eigensinnig sein will und dennoch ihren Schöpfer ernähren soll, bestand von Anfang an ein unaufhebbarer Widerspruch.

Gottfried Semper hatte schon 1860 die in der Moderne ausdifferenzierten Techniken, Handwerke und Künste aus frühgeschichtlichen Kulturtechniken rekonstruiert (Semper 1860/63 und 1852). Hier ist das Konzept der angelsächsischen arts \& crafts-Bewegung, des Jugendstils und des Deutschen Werkbunds, aber auch des Bauhaus vorbereitet. In anderer Weise realisierte Aby Warburg solche Konzepte, wenn er eine Kulturwissenschaft begründete, welche eine Briefmarke derselben analytischen Sorgfalt unterzog wie die Fresken des Palazzo Schifanoia in Ferrara.

Hannah Arendt benutzte gern ein antikes Motto, das uns an Rousseau erinnert. Es lautet: "Niemals ist man tätiger, als wenn man dem äußeren Anschein nach nichts tut, niemals ist man weniger allein, als wenn man in der Einsamkeit mit sich allein ist." (Arendt 1981: 317; s. auch Arendt 1998: 10) $)^{5}$ Dies ist ein Zitat aus Ciceros De re publica, wo Cato den Satz als Ausspruch von Scipio Africanus überliefert, der ein höchst dynamischer Feldherr und Staatsmann war. Und gerade er formuliert einen Satz, der jenen ziel- und nutzlosen Zustand im Rousseauschen Boot als die höchste Tätigkeit darstellt. Das scheinbar Nutzlose als höchste Tätigkeit und das scheinbare Asoziale als höchste Form der Sozialität! Man versteht nun, was die Spannweite der Kultur ausmacht, die wir Kulturwissenschaftler zu untersuchen haben.

Wir müssen immer wieder neu vertreten, dass die Kategorie des Nutzens nicht von der einen, ökonomischen Form des Kapitals abhängt, sondern dass es, wie Pierre Bourdieu betont, auch das symbolische und das kulturelle Kapital gibt (Bourdieu 1983 und 1987). Dessen Werte aber sind für die Selbsterhaltung und Entwicklung der Gesellschaft essentiell.

Setzen wir also voraus, dass das Schweifende und Nutzlose, dass die Tätigkeiten also der Einbildungskraft, die nichts will, als sich zu verkörpern, die Sphäre beschreibt, in der die Künste gedei-

5 Das Zitat von Cato wurde verwendet von Söllner 1990: 224, später auch in: Söllner 1996: 247; übernommen von Han 2009: 104f.; vgl. auch Geisen 2011: 288. 
hen, aber auch die Arbeitsgesellschaft und ihre Pathologien wachsen.

\section{Arbeitsgesellschaft und Erschöpfung}

Der 24-Stunden-Rhythmus der Moderne, ihre niemals erlahmende Dynamik und Mobilität, das ununterbrochene Strömen der Energien, Kräfte, Massen und Dinge, die Unruhe und Geschwindigkeit, die sich am konzentriertesten in der Megalopole und in den Fabriken darstellten, bezeichnen eine Gesellschaft ohne Ermüdung und Erschöpfung. Müdigkeit ist ein unerwünschtes survival des vormodernen Körpers, der sich erholen muss, um wieder aufs Niveau der Maschinen zu kommen, die das Maß aller Dinge sind. Kein Wunder, dass in dieser Zeit die Thermodynamik und mit ihr eine gleichsam entropische Stimmung aufkam (Neswald 2006). Anson Rabinbach (1992) beschreibt, dass der ideale Grenzfall der Arbeitsphysiologie des 19. Jahrhunderts der ermüdungsfreie Organismus war: Er stünde auf einer Höhe mit den Maschinen. Doch die Großstadt ohne Schlaf und die Fabrik ohne Pause fanden ihren Gegenspieler in der ungeheuren Müdigkeit, die sich im Herzen der Industriegesellschaft ausbreitete: als Entfremdung, Neurasthenie, chronische Erschöpfung, Depression. Von Marx bis Freud breitete sich ein riesiges Diskursfeld über die Sozialpathologien der urbanen Industriegesellschaft aus.

All das glauben wir heute wiederzuerkennen, wenn wir mit Byung-Chul Han (2010) von der Müdigkeitsgesellschaft sprechen ${ }^{6}$ : Die neurasthenischen Symptome wiederholen sich in den endemischen Depressionen und Burnouts, denen man damals wie heute mit Sport und Lebensreform, aber auch mit Alkohol und Drogen, mit Psychopharmaka und Psychotherapie begegnet. Gewiss wird angesichts dieser peinigenden Müdigkeit auch eine inspirierende Müdigkeit entdeckt, so bei Peter Handke - eine Müdigkeit der negativen Potenz, die das quälende "Um-Zu" allen Handelns in ein ästhetisches "Nicht-zu" (Handke 1989: 62) verwandelt. Nichts darf sein, was nicht eine Funktion hat; doch alles, was funktionslos ist wie diese herrliche Müdigkeit, hat Wert. Peter Handke

6 Vgl. auch Ehrenberg (2004); Roelcke 1999. schreibt: "Die Inspiration der Müdigkeit sagt weniger, was zu tun ist, als was gelassen werden kann. [...] Die Müdigkeit als das Mehr des weniger Ich." Müdigkeit als Kunst des Gelassen-Seins (ebd., 74f.). ${ }^{7}$

Durchaus gilt, dass in solchen Kontrapunkten zur Arbeitsgesellschaft ein rousseauistisches Erbe nachwirkt. Seit Rousseau datiert der Diskurs, dass die fortschreitende Kultur einen unerwarteten Preis kostet: Sie schlägt auf die Gesundheit und Natürlichkeit des Menschen zurück. Und diese Denaturierung wird zum Kern des Diskurses über Degeneration und später über Dekadenz als Folgen der Zivilisation. ${ }^{8}$ Die Kulturkritik gehört zu den Attitüden der Selbstbeobachtung des Bürgertums, das der Antreiber eben jenes technischen Fortschritts und jener großstädtischen Lebensformen ist, unter denen es dann zu leiden beginnt.

Die Rousseausche Linie wurde vor allem in der Romantik aufgenommen. Auch das kategoriale Inventar der zivilisationskritischen Diagnosen, nämlich das Paradigma der Nerven ${ }^{9}$ und das mit inm fusionierte Modell der Elektrizität, sind in der Medizin mit der romantischen Ästhetik und Erzählkunst gekoppelt. Erschöpfende Arbeitswelt und strenge Pflichtethik treten erstmals zu erfüllter Muße und arbeitsloser Phantasieaktivität in einen Widerspruch. Dieser wird im Lauf des 19. Jahrhunderts zum Epochenwiderspruch der bürgerlichen Gesellschaft. Viele Leitdifferenzen entspringen hier: Erfüllung versus Entfremdung, Muße versus Arbeit, Lust versus Pflicht, Liebe/ Leidenschaft versus Ehe/ Familie, Künstlerdasein versus Berufsleben, volle, ereignishafte versus leere, repetitive Zeit, Phantasie versus Verstand usw. Von solchen Dualismen wurden auch die medizinischen Diskurse bestimmt. Je nach Position des Autors wurden die ruhelose Zivilisation und drückende Arbeitslast für die Psychopathologien verantwortlich gemacht, oder umgekehrt wurden die Künstler, Außenseiter, Bohemiens,

7 Zu denken ist hier auch an Heideggers (1994) Plädoyer für die Gelassenheit (hätte er diese 1933 doch gewahrt!); vgl. Voigt/Meck 2005; Hobuß/Tams 2014.

8 Beispielhaft nenne ich: Nordau (2012). Viele Stellen bei Nietzsche wären zu nennen. - Forschungsliteratur dazu z.B.: Drost (1986); Pick (1993); Constable/Denisoff/Potolsky (1998); Pross (2013); Mahoney (2015); Sherry (2015).

9 Grundlegend zum ,nervösen Zeitalter': Radkau (1994) und (1998). 
Flaneure, Arbeitsverweigerer, Alkoholiker als degenerierte oder dekadente Subjekte stigmatisiert.

Auch Nietzsche wendet sich gegen solche "Verkündiger der grossen Müdigkeit".10 Für inn ist diese dekadent, eine "andere Welt", die ein "Synonym des Nicht-seins, des Nicht-lebens, des Nicht-leben-wollens" ist ${ }^{11}$, "die gefährlichste und unheimlichste Form aller möglichen Formen eines ,Willens zum Untergang', zum Mindesten ein Zeichen tiefster Erkrankung, Müdigkeit, Missmuthigkeit, Erschöpfung, Verarmung an Leben".12 Müdigkeit, Erschöpfung und Dekadenz sind bei Nietzsche fast austauschbar. Wenn früher die Religion die „zur Epidemie gewordnen Müdigkeit und Schwere"13 bekämpfte, so sind es in der industriellen Arbeitsgesellschaft die Medizin und Psychiatrie. Freche Pamphlete wie "La droit a la paresse" (1883) von Paul Lafargue (1966) waren hingegen ein Anschlag auf die Primärtugenden der Gesellschaft, zu denen nicht das Recht, sondern die Pflicht zur Arbeit gehörte.

Man muss sich klar machen, dass Müdigkeit und Erschöpfung zwar zu allen Zeiten als Phänomene vorkommen. Ihr Aufstieg indes zur Epochensignatur zeigt in der Geschichte der physischen Befindlichkeiten, Emotionen und Mentalitäten dennoch eine historische Besonderheit. Das Zeitalter als eines der Nerven zu bezeichnen, ist historisch singulär. Für die zeitgenössischen Beobachter schwankte die Lebenskraft permanent zwischen Überspannung und Erschlaffung, Überreizung und Reizarmut. Nichts war so sehr ein Resonanzraum der Erregungen und Stimmungen, der zivilisatorischen Anstrengungen und Vergnügungen wie das System der Nerven, die seit 1800 eine beispiellose Karriere gemacht hatten. Die Nerven reagierten sensibel oder irritabel auf die endogenen Prozesse von Körper und Seele ebenso wie auf die exogenen Impulse der Zivilisation. Sie waren wie nichts anderes geeignet, zur Leitmetapher der Zivilisationskrankhei-

10 Nietzsche (1988): Also sprach Zarathustra, KSA IV: 300.

11 Nietzsche (1988): Nachgelassene Fragmente Frühjahr 1888, KSA XIII: 354.

12 Nietzsche (1988): Versuch einer Selbstkritik - Die Geburt der Tragödie, KSA I: 18/9 (als Kritik an der christliche Kultur).

13 Nietzsche (1988): Zur Genealogie der Moral, KSA V: 378. ten und damit zum Titel der Zivilisation selbst zu werden. Es ging nicht mehr um die Trägheit der notorischen Faulpelze, Vagabunden, Armen, Bettler, die Überarbeitung der gepressten Bauern, um Furcht und Zittern der armen Söldner, um die acedia der Mönche und die Melancholia der Gelehrten, nicht mehr um die blasierten Müdigkeiten der gelangweilten Hofadligen, nicht um die Desperatio hochbegabter bürgerlicher Hofmeister oder die Erschöpfung zügelloser Libertins. Diese alteuropäischen Müdigkeiten trugen eine andere soziale Physiognomie, erfuhren andere, nämlich metaphysische, moralische oder christliche Deutungen - oder sie versanken im Bauch der Geschichte, eine nicht-signifikative Masse, in deren Stummheit sich Müdigkeit und Erschöpfung vermummt hatten. Gerade diese Sphären gehören zu den Arbeitsfeldern der Kulturwissenschaft.

Die moderne Müdigkeit ist im Kern ein Element der hochorganisierten Arbeit. „Die endemische Unordnung der Ermüdung - die augenscheinlichste und hartnäckigste Mahnung an den halsstarrigen Widerstand des Körpers gegenüber unbegrenztem Fortschritt und Produktivität - begleitete die Entdeckung von Krafterhaltung und Entropie. Die Ermüdung wurde zur permanenten Nemesis eines Europas der Industrialisierung." (Rabinbach 1992: 14) Mit der Ermüdung fand erstmals eine negative Kraft Eingang in die Selbstbeschreibung der Moderne: die Abwesenheit von Frische, Aufmerksamkeit, Spannung, Aktivität, Kraft, Produktion. ${ }^{14}$ Diese ließen sich nur von ihren Grenzen in der Ermüdung her verstehen, ja, die Fortschrittsaspirationen des Jahrhunderts kippten sogar ins Gegenteil: Die entropische Müdigkeit, die Neurasthenie sind der Normalzustand gegenüber den Phasen manischer Arbeit, welche die Müdigkeit immer nur unterbrechen. Man lese George Miller Beards American Nervousness (1881; s. auch Beard 1880), den Erfinder der Neurasthenie, dessen Buch blitzschnell in ganz Europa sich verbreitete und den Diskurs der Ärzte, Psychiater und Kulturkritiker bestimmte. Ähnlich einflussreich war die empiri-

14 Mit Deleuze kann man sagen: „Der Ermüdete verfügt über keinerlei subjektive Möglichkeit mehr, er kann also gar keine objektive Möglichkeit mehr verwirklichen. [...] Der Ermüdete hat nur ihre Verwirklichung erschöpft, während der Erschöpfte alles, was möglich ist, erschöpft." (Deleuze 1996: 51). 
sche Arbeit von Angelo Mosso (1892; vgl. dazu Felsch 2006).

Angesichts dieser Zeitstimmung sind nicht mehr Subjekte müde und erschöpft, sondern die Welt drängt ihrer finalen Erschöpfung zu, wenn sie nicht bereits eingetreten ist. Das ist die Stimmung der Neurastheniker um 1900: "Ganz vergessener Völker Müdigkeiten / Kann ich nicht abtun von meinen Lidern", heißt es bei Hofmannsthal 1895 (Hofmannstahl 1979: 26). Finis hominis! Posthistoire, Postmoderne schon hier!

Die Robustheit der Industrie und der Technik, die Rationalität und die Wissenschaften, das Wachstum der Städte und des Verkehrs, die Macht von Staat und Militär, die Innovationskraft der Medien und die Verschaltung von allen und allem durch Netzwerke und logistische Infrastrukturen bildeten die unwiderstehliche und dynamische Mitte der Gesellschaft des langen 19. Jahrhunderts, das bis 1914 andauerte. Doch diese Mitte wurde ständig von den Rändern her heimgesucht: durch Alkoholismus, Degeneration, Dekadenz, Neurasthenie, Hysterie, Psychosen, Nervenkrankheiten, durch soziale Verwahrlosung und ,moralischen bzw. physiologischen Schwachsinn [des Weibes]' (moral insanity; Paul Julis Möbius; Katinka von Rosen), „Entartung" (Max Nordau) und Kriminalität (Cesare Lombroso), durch Überreizung oder Reizarmut, Spiritismus und Okkultismus, durch krankmachende Geschwindigkeit sowie durch endemische Krankheiten wie Cholera, Tuberkulose, Typhus, Bleichsucht, Syphilis u.a.m. Dieser fürs 19. Jahrhundert eigentümliche Widerspruch aus stämmiger Robustheit und müder Schwäche ließ, als Moment der Moderne, die Kulturkritik entstehen und mit ihr die Reflexivität und Dialektik, wie sie für die Moderne ebenso kennzeichnend sind wie Technik und Industrie. Ja, man kann sagen, dass es niemals zuvor eine Epoche gegeben hat, die in eins mit ihren unbestreitbaren Fortschritten und Gewinnen zugleich die eigenen Sozialpathologien und gesundheitlichen Kosten so radikal reflektierte wie das fin de siècle. In vieler Hinsicht ist die Neurasthenie vielleicht weniger eine wirkliche Krankheit, als der genau rechtzeitig erfundene Spiegel, worin die triumphierende Arbeitsgesellschaft ihr erschöpftes Antlitz studieren konnte. In anderer Hinsicht ist die Neurasthenie eine unbestimmte, alle möglichen Symptomatiken inkludierende, weder nur physische noch nur psychische, weder nur neuro- logische noch nur soziale Krankheit, doch irgendwie alles zusammen. Wenn man im Warenhaus die glitzernde Schauseite der Warengesellschaft erblicken konnte (wie Émile Zola in Au Bonheur des Dames [1884] schildert), dann darf man die Neurasthenie geradezu als den Kramladen für die odds and ends des 19. Jahrhunderts bezeichnen. Die schön verpackten Frauen und literarischen Elegants, in Salons und auf Boulevards gleichermaßen zu Hause, mit müden Gesten und feinsinnigen Bonmots, in bleicher Schönheit, gelagert in weichen Fauteuils, die Huldigungen der Männer und die besorgten Blicke der Ärzte hinnehmend - und auf der andere Seite die ausgelaugte Fabrikarbeiterin, bleichsüchtig mit fünf Kindern und einem aggressiven Alkoholiker in einer lichtlosen Zweizimmerwohnung lebend, und die jungen Burschen, die kraftlos ihrem stundenweise vermieteten Schlafplatz entgegenwanken: Sie alle sind, mit Hermann Bang (1857-1912) zu sprechen, "Hoffnungslose Geschlechter" (1880, dt. 1900).

Es ist durchsichtig, dass eine eher konservative Moderne-Kritik sich mit der Medizin verbündet, um dem chaotischen Diskurs Gewicht und Ansehen zu verschaffen. Es ist aber auch klar, dass in den Sammelsurien von Symptomen sich diffuse Modernisierungsschäden Ausdruck und Beachtung verschafften, die in der Tat ernst zu nehmen waren, auch wenn sie in der ideologischen Fusion mit Theorien über allgemeine Degeneration, Dekadenz und - so bei Max Nordau - Entartung für heutige Leser schwer erträglich sind. Der medizinische Kern ist weitgehend identisch mit dem der Ermüdungswissenschaft: Der aufgrund der Modernisierung aller Lebensverhältnisse enorm angestiegene Verbrauch an Nervenkraft führt zu einer allgemeinen Erschöpfung der psychophysischen Substanz des Individuums, aber auch des Kollektivkörpers der Gesellschaft. Die gesellschaftlichen Leiden führen zum Leiden an der Gesellschaft (H.P. Dreitzel 1968). Man ist müde, man ist der Gesellschaft müde, man ist des Lebens müde. Die Mikro- und die Makroebene wurden im Neurasthenie-Diskurs zusammengeschaltet, so dass schließlich die erschlafften Subjekte sich im Gleichklang mit der Ermattung und Erschöpfung der ganzen Epoche befanden. Auch wenn die Terminologie und die wissenschaftlichen Ansprüche andere geworden sind: Die zirkulierenden Diskurse über den Stress, das Burnout 
und die endemischen Depressionen heute laufen im Prinzip noch immer auf der kulturkritischen Schiene, auf welche der Neurasthenie-Diskurs so erfolgreich Fahrt aufgenommen hatte. Wir sind krank, weil die Gesellschaft krank ist: Das ist ein noch immer funktionierendes Erfolgsrezept der Kulturkritik.

\section{Risiko und Sicherheits- Dilemmata in der gegenwärtigen Gesellschaft ${ }^{15}$}

Seit der Aufklärung gehört es zum Selbstverständnis moderner Gesellschaften, dass die Rationalisierung der Welt Gewissheiten generiert, welche die Ordnungen des menschlichen Lebenszyklus, der Staaten und der Gesellschaft stabilisieren sollen. Die kognitiven Ordnungen und gouvernementalen Regimes, welche die Transformation traditionaler in funktional ausdifferenzierte Gesellschaften antrieben, erhöhten jedoch nicht nur den Standard staatlicher Sicherungssysteme, sondern gleichzeitig die Kontingenz. Kontingenz meint, dass Angst und Gefahr, Zufall und Unordnung, Katastrophe und Unglück, Biographie und Lebensformen, Erfolg und Zufriedenheit nicht mehr durch unverfügbare Ordnungen gerahmt sind. Im Ergebnis führte diese, Entbettung' für die Individuen zu massiven Überlastungen. Trotz gewachsener regierungstechnologischer Potentiale waren strukturelle Paradoxien und unsteuerbare Zyklen von Aufschwung und Depression die Folge.

Moderne Gesellschaften müssen ihre Identität auf riskanten Wandel, auf Bewegung, Zerstörung und Wachstum einstellen. Unsicherheit ist ihre Entwicklungsvoraussetzung. Aber der Innovationsdruck in Kombination mit der Enttraditionalisierung bedeutet für viele Menschen nur noch Stress. Und so erwächst aus dem Modernisierungsmodus des Möglichkeitsdenkens eine Nachfrage nach Bewältigungsformen dieser Verunsi-

15 Zum Folgenden vgl. aus politikwissenschaftlicher Sicht: Münkler et al. (2010); Münkler et. al (2010a): Handeln unter Risiko. Gestaltungsansätze zwischen Wagnis und Vorsorge. In die Diskussion gebracht wurde das Thema schon zuvor: Beck (1987), Beck (2007) und Luhmann (1991). Kulturhistorisch: Bernstein (1997); Hampe (2006). cherungen. Dies drückt sich, in Zeiten wachsender Entstaatlichung der Daseinsvorsorge, auch in der Zunahme zivilgesellschaftlicher Dienstleistungen aus, die von privater Altersvorsorge bis zu Psychotherapie oder Fitnesstraining reichen. Und dazu gehört auch die Kultur, die angesichts der Modernisierungsschäden verstärkt nachgefragt wird, freilich nicht mehr als bildungsbürgerliche Hochkultur, sondern als Spektakel, das längst auch die Opernhäuser und Kunstfestivals erreicht hat.

Die in der Moderne aufgebauten Einrichtungen der Sekurität generierten ein Lebensgefühl, das nicht mehr in religiöser Selbstvergewisserung, sondern in ökonomisch verrechneten Garantien wurzelte. Diese wurden freilich erkauft mit dem Bewusstsein um die Zufälligkeit des eigenen Handelns und um die Unzuverlässigkeit der staatlichen Institutionen: An die Stelle religiöser Heilsgarantien trat ein Risiko-Management, das der Staat für die Gesellschaft und der Bürger für sein Lebensskript zu entwickeln hatte. Genau diese Strategien und Versprechen brechen heute zusammen. Dies führt zu einer befremdlichen Diagnose: Die Moderne stellt die Erweiterung des Möglichkeitsraums auf Dauer, während die Mentalitäten nicht in gleicher Weise mitgewachsen sind. Es verbreiten sich misstrauische, ungläubige, depressive Stimmungen. Dies ist die Vertigo-Moderne, der wir soeben beiwohnen. In traditionalen Gesellschaften war die Religion die zentrale Institution für Sinnstiftung. Ökonomie war Heils-Ökonomie: für die Tröstung bei innerweltlichen wie metaphysischen Ängsten, für die Bewältigung des allgegenwärtigen Todes und für die Vermittlung von ,Geborgenheit' im Schoß einer Zeit, die jeden Einzelnen in das Heilshandeln Gottes zwischen Ursprungsereignis und Endgericht einhegte. Die metaphysische Rahmenlosigkeit der Moderne hat zwar die Spielräume der Kontingenz und damit die Räume selbstregulierter Gestaltung ständig anwachsen lassen. Doch zugleich wurden den Instanzen, die diesen Prozess vorantrieben, die Erwartung aufgebürdet, die drohende Sinnleere, die Unsicherheit und Zukunftsungewissheit, den psychophysischen Stress in einer Wettbewerbsgesellschaft nicht nur zu beruhigen, sondern in planbare Lebensläufe und in wohlfahrtsstaatliche Garantien zu transformieren. Heute aber sind weder Lebensläufe planbar noch ist auf staatliche Fürsorgemaßnahmen Verlass. Erwartungsüber- 
lastung auf der einen, Erwartungsenttäuschung auf der anderen Seite erzeugen eine Art Lähmung des für die Moderne unerlässlichen Möglichkeitssinns. Der Effekt ist: Die risikoaffine Dynamik der Moderne ist eigentümlich mit risikoaversen Mentalitäten verkoppelt.

Die Moderne hat anhaltende Schwierigkeiten damit, die Prozesse ständiger Verflüssigung und Veränderung, der Innovation und des Experimentierens auszubalancieren durch Mechanismen der sozialen und politischen Stabilität oder durch Sinnsicherung und Traditionsbildung. Das Risikomaß, das einen Vorsprung im Wettbewerb verspricht, ist nicht beliebig zu erhöhen, wenn es keinen Gegenhalt in wirksamen Stabilitätsmechanismen auf individueller wie gesellschaftlicher Ebene gibt. Das bedeutet: Risiko und Sicherheit sind nicht nur komplementär, sondern auch proportional. Wächst das Risiko, muss Sicherheit mitwachsen; werden bestimmte Niveaus von Sicherheit unterschritten, lässt die Risikobereitschaft nach.

Die Frage nach Sicherheit und Risiko ist eine Schlüsselfrage moderner Gesellschaften. ${ }^{16}$ Der Zwang zu Beobachtung, Forschung und Reflexion hat sich angesichts des jüngsten Finanzcrash dramatisch erhöht. Unklar aber ist, wer die neuen Eliten bilden könnte, nachdem die alten blamiert sind. So kann man auf der einen Seite, bei den Globalisierungsgewinnern, eine Zunahme experimenteller, ja extremer Risikobereitschaft identifizieren, während auf der anderen Seite Konformismus, larvierte Wut und Depression oder als Amüsement getarnte Langeweile grassieren. Von beiden Seiten ist nichts zu erwarten, zumal Risikokompetenz und Sicherheitsbedürfnis eklatant asymmetrisch verteilt sind. Sie differenzieren sich zu Stilen des Lebens aus - und reißen die Gesellschaft noch stärker auseinander, nicht nur ökonomisch, sondern auch mental, soziokulturell und lebensweltlich. Denn es gibt Verlierer und Gewinner des Modernisierungsspiels. Es ist deutlich, dass moderne Gesellschaften den Risiko-Habitus belohnen - den Börsenmakler wie den Wissenschaftler in Risikozonen, den global player wie den Kulturscout -, während es für die Ängstlichen und Gehemmten kaum mehr gibt als die illusionäre Teilhabe an Twitter, Face-

16 Zum Standard geworden sind Beck (1987) und Beck (2007). book oder Fernsehen. Banker und Börsenmakler aber sind die Nihilisten unserer Tage, die ihr Schäfchen längst im Trocknen haben, wenn die Träume der Schafherde sich in Nichts auflösen. Die modernen Nihilisten sind, mit Umberto Eco, die „Apokalyptiker"17, für die der Untergang zum Risiko gehört, das heute jeder einzugehen hat, der mitspielen will. In dieser Welt, in der es keine Moral gibt, interessiert nicht die Frage danach, welches Unglück angerichtet wird, sondern nur die Frage, wann der Zeitpunkt zum Ausstieg gekommen ist. Den kennen die "Integrierten" nie. Ihnen, die auf Ruhe und Ordnung setzen, und damit ungewollt zur Vermehrung der Risiken beitragen, bleibt oft nur die scheinheilige Empörung, dass die Welt ,schlecht' ist und die master of the universe nichts als kriminelle Spieler sind. Gerade mit dieser Mentalität stützen sie den apokalyptischen Diskurs, von dem der Risiko-Thrill der anderen seine Renditen und Boni bezieht.

Die Standardposition der Moderne, nämlich Fortschritt durch Erhöhung von Sicherheit zu stabilisieren, ist in Frage gestellt. Es ist unwahrscheinlich, dass Antworten auf die beschriebenen Problemzonen nur politologisch, soziologisch und ökonomisch gefunden werden können. Gefragt sind kultur- und religions-historische Forschungen, welche die Tiefendimension der Sicherheitsund Risikoparadoxien der Moderne erforschen. Dies könnte auch ein Beitrag zur Unvermeidlichkeit der Kulturwissenschaft sein.

\section{Kulturelle Diffusion des Religiösen}

Die westlichen Demokratien haben darauf verzichtet, normative Zentren oder sinnhafte Rahmen auszubilden; sie setzen statt dessen auf formale Verfahren der Konfliktregulierung von Systemebenen und Populationen, die zu vereinheitlichen niemand sich mehr zutraut. Im Gegenteil wäre ein solcher Versuch unvereinbar mit den säkularen Prinzipien, auf die unsere Gesellschaften verpflichtet sind. Die Folge hiervon ist jedoch ein krasser Mangel an gesellschaftlicher Synthesis. Dieser Mangel wird ausgependelt durch öko-

17 Die Typik Apokalyptiker versus Integrierte entnehme ich: Eco (1985). 
nomische Effizienz, politische Hegemonie und notfalls durch militärische Intervention, wie dies an den USA zu beobachten ist.

Man kann den Religions- und Weltbild-Verzicht die andere Seite eines wild wuchernden Orientierungsbedarfs nennen. Säkularisierung und Wiederverzauberung sind zwei Seiten derselben Medaille. Die widerspruchsvollen Konstellationen, die Religion und Moderne dabei eingehen, bilden auch den Hintergrund dafür, dass Sport oder Musik zu einer gewaltigen Pop-Industrie geworden sind: Die ehemals durch die Großreligionen gebundenen religiösen Energien flottieren durch alle Systemebenen und stopfen die Sinn- und Erlebnisleere, die durch Verfahrensrationalität und formale Demokratie entstanden ist. Gerade Kirchen vermögen solche Erlebnisevidenzen nicht mehr herzustellen - es sei denn, sie agieren im Muster moderner Pop-Kultur. Guy Debord (1967) sprach schon von der Gesellschaft des Spektakels. ${ }^{18}$ Dies bestimmt weite Bereiche von Politik oder der Kunst, des Rechts und sogar der Börse. In den spektakulären Performances werden die semantischen Ressourcen und der soziale Sinn einer Kultur erst erzeugt. Dabei werden popkulturelle Großkunstwerke, wie der Fußball eines ist, zu einem grandiosen patchwork, das sich aus den religiösen Überlieferungen beliebiger Kulturen bedient.

Ich möchte Ihre Aufmerksamkeit aber lenken auf ein befremdliches Phänomen: Die Moderne ist keineswegs nur funktional differenziert, sondern sie weist ein parasitäres Verhältnis zu überlieferten Formen der Religiosität auf. Meine These ist: Politik und das Alltagsleben, sogar die Wissenschaften, die Medien, der Sport, die Event-Kultur und die Künste zehren von toten und lebenden Elementen der Religionen. Ich lasse offen, ob es sich hierbei um Auflösungs- oder Transformationsprozesse des Religiösen handelt. Mein Befund ist: das Religiöse ist auch in modernen Kulturen ubiquitär. Die Moderne indes, so die weitere These, durchschaut wiederum nicht ihre religiösen Anleihen und weist darum eine unbegriffene Ambivalenz von Säkularisierung und Verzauberung auf.

Gewiss haben die Religionen des Westens ihre Fähigkeit eingebüßt, das alltägliche Leben großer

$18 \mathrm{Vgl}$. zum Folgenden auch: Böhme (2002) und Böhme (2006).
Massen zu strukturieren, ihm Sinn zu verleihen und es mit transzendierenden Perspektiven zu bereichern. Es zeichnet sich zudem ab, dass die Fragen der religiösen Differenz nicht länger unter religiösen, sondern zunehmend unter Gesichtspunkten der Ethnizität, der Multikulturalität, der Migration und der Globalisierung behandelt werden. Das aber heißt: Religionen werden kulturell beurteilt, und nicht umgekehrt Kulturen religiös.

Damit allerdings werden sozialer Sinn und transzendierende Ereignisse kontingent: Je nach Bedarf werden sinnintensive Gemeinschaftserfahrungen durch Papstbesuche, Festivals oder Fußball-WM inszeniert. Oder sie werden subkulturell ausdifferenziert, z.B. nach multiethnischen Minoritäten (Karneval der Kulturen in Berlin), sexueller Orientierung (Christopher Street Parade) oder Altersklassen (Festivals des Deutschen Seniorentags oder der katholischen Jugend).

Der Verlust sozialer Synthesis einerseits sowie die Zunahme an Kontingenz andererseits haben den paradoxen Effekt, dass das Religiöse die Form flottierender Energien annimmt, die alles und jedes mit willkürlicher Wucht und mächtigen Bindungskräften besetzen können. Dies sind religiöse Phänomene einer wilden, weil kaum demokratisch rückgebundenen Energie und einer heiBen, weil nicht reflexiv kontrollierten Dynamik, die es wahrscheinlich machen, dass sich auch die westlichen Kulturen in eine Mixtur religiöser Energien verwandeln.

Vergleichbar geht es in der Welt unserer aufgeklärten Staatsbürger zu: funktionale Effizienz unter der Woche, kollektive Ekstasen auf KulturEvents am Wochenende; rationale Zukunftssicherung hier und Suche nach Risiko-Thrill dort; ökonomischer Kalkül einerseits und andererseits Übernahmen aus exotischen Kulturen; Teilnahme an demokratischen Prozessen und zugleich quasireligiöses Aufgehen in ,Gemeinschaftskörpern' des Sports und des Entertainment. Diese widersprüchlichen Mischungen treten dabei nicht nur als Handlungsmuster von Gruppen auf, sondern sie wandern in die Subjekte selbst ein. Der massenmediale Star-Kult zieht ins Parlament, die Gnosis ins Internet ein; der Kapitalismus funktioniert nur unter Zuhilfenahme fetischistischer Formen; der Sport arbeitet in Formen ,mystischer Partizipation'; Festivals entleihen ihre Attraktivität der vergangenen Kraft von Mysterien; das gesellschaftlich Imaginäre wird - in Tausenden 
von Filmen - von den Monstrositäten aller Zeiten bevölkert; ,Gott ist tot' bildet nicht den Übergang zu einer säkularen Gesellschaft, sondern zum Erwachen von Abertausenden neuer Götter.

Wichtige soziale Integrationen der Moderne laufen mithin über Mechanismen, welche eben diese Moderne als nicht zu ihr gehörig, als vormodern und irrational abtut. Darin steckt auch eine Gefahr: Die Moderne ist zu ihrem Erhalt auf ihr Gegenteil angewiesen. Die Zivilgesellschaft hat das Irrationale, ja Barbarische nicht hinter ihrem Rücken, sondern womöglich in sich selbst. Nichts scheint falscher zu sein als die These von der Entzauberung der Welt. Die flottierenden Religionsformen heute belehren im Gegenteil darüber, dass die Entzauberung im Namen der Rationalität zu einem Schub von Wiederverzauberung geführt hat.

\section{Perspektiven der Kulturwissenschaft}

Im Kontext klassischer Disziplinen fällt die Schwierigkeit auf, Methoden, Theorien und Gegenstände der Kulturwissenschaft bestimmen zu können. ${ }^{19}$ Was ist ihre differentia specifica? Wenn man Kulturwissenschaft als Fach vertreten will, muss man Handicaps hinnehmen, was ihre schwache Definiertheit angeht; man muss ihre vielen Überschneidungen mit anderen Fächern akzeptieren - und zugleich muss man verteidigen, dass sie kein Fach wie andere ist; man muss demonstrieren können, dass dasjenige, was ihre Schwächen ausmacht, zugleich ihre Stärken sind, dass inr Risiko zugleich das ist, was ihr interdisziplinäre Kompetenz ausmacht.

Eine historische Kulturwissenschaft versteht ihre Rolle vor allem darin, die langwellige Entstehung europäischer Kulturen, aber auch ihres Zugs zur Globalisierung zu untersuchen. Dabei geht sie davon aus, dass ,Europa' historisch längst realisiert war. Das sog. lateinische Mittelalter ist eine Form transregionaler Europäisierung von religiösen, politischen, sozialen und kulturellen, aber auch wissenschaftlichen und künstlerischen Pro-

19 Zum Folgenden vgl. etwa Conermann (2012); Leggewie/ Lang/Zifonun (2010); Allerkamp/Raulet (2010); Kusber (2010); Csáky/Leitgeb (2009); Wirth (2008). zessen, die miteinander in vitaler Wechselwirkung und dichter Vernetzung standen. Von der frühen Neuzeit an bildet sich ein neuartiges, europäisch funktionierendes Netz der Wissenschaften und der Medien aus. Die Entstehung der modernen (National-)Staaten ist mit einer binneneuropäischen politischen Verflechtung und Konkurrenz ebenso verbunden wie mit der imperialen Ausweitung europäischer Kulturformen im globalen Maßstab. In den Künsten, den Wissenschaften, der Aufklärung und den neuen sozialen Bewegungen entstehen, teilweise im Schutz, teilweise in Opposition zur staatlichen Macht, transnationale Geflechte der Kommunikation und des Austauschs, die von europäischen Ideen inspiriert sind. Insbesondere erweisen sich die Künste und die Techniken als Motoren übernationaler, ,europäischer' Praktiken und kultureller Leitbilder. In dieser longue durée ist Kulturwissenschaft zu situieren.

Die schneller auf kulturelle und politische Veränderungen reagierenden Humanities in den USA sind bekanntlich stark durch politische Faktoren bestimmt worden (Bachmann-Medick 2006; vgl. Böhme 2008). Dazu gehörten so verschiedene Vorgänge wie die im Zuge des Postkolonialismus selbstbewusste Visibilisierung unterdrückter regionaler, ethnischer und religiöser Überlieferungen und Minoritäten; die neuen Verteilungen von Populationen, Gütern und Dienstleistungen unter Bedingungen polyzentrischer und zugleich hegemonialer Globalisierung, die ihrerseits neue Formen von Regionalisierung, Lokalisierung und Hybridisierung hervorbringt; die Entortung ganzer Bevölkerungsteile in den weltweiten Migrationsund Flüchtlingsströmen; die Verschiebung, Auslöschung und Kreation von Zentren wie Peripherien der Macht; die globale Verbreitung von Popkultur und Warenästhetik bei gleichzeitig wachsenden globalisierungskritischen Widerständen; die Entstehung transnationaler communities durch neue Kommunikationstechnologien. Damit verbunden waren Krisen der westlichen Formen der Repräsentation kultureller Werte, Normen und Wissensordnungen, die in ihren kolonialistischen Gesten dekonstruiert wurden. Verbunden damit spielten policies of sex, gender and race eine bedeutende Rolle und schlugen auf der Ebene universitärer Wissenschaften durch. Solche Prozesse sind es, die zu den Transformationen tradierter Disziplinen im Zeichen der cultural turns sowie zu einer Fülle von Neugründungen von Fächern oder studies 
geführt haben. In den USA war es nicht anders als bei den englischen cultural studies, die ohne den politischen Hintergrund der proletarischen Kultur nicht verständlich wären.

Gerade bei den cultural turns wird sichtbar, dass die wissenschaftliche Ethik, die Verfahren, die Kategorien und sogar die Theorien von den ,Gegenständen' mitbestimmt bleiben, denen sich die turns verdanken. Viel mehr als andere wissenschaftliche Modelle tragen die cultural turns einen politischen Index, den abzustreifen sie um das bringen würde, was sie erklärter Maßen sein wollen: nicht nur explorierende und explanative, sondern eingreifende Wissenschaft. So verspricht die kulturwissenschaftliche Wende neben Differenzierungszuwächsen vor allem moralische und politische Gewinne: eine bessere Praxis in den Feldern des Multikulturalität, der Minoritätenpolitik, des Euro- und Logozentrismus, der latent oder offen kolonialistischen Hierarchisierung von Kulturen usw. Es wäre aber ein Irrtum anzunehmen, dass die Kulturwissenschaft mit den Feldern gleichzusetzen wäre, die in den cultural turns ins Spiel gebracht wurden.

Lassen Sie mich festhalten: Bezogen auf Fragen der Medien, des Geschlechts, der Kultur sind manche Universitäten den Weg gegangen, diese ,Felder' als Querschnittsorientierungen zu empfehlen, während andere Universitäten es bevorzugten, neue Fächer einzurichten: Medienwissenschaft, Kulturwissenschaft, Gender Studies. Doch bedeutet die Entscheidung für ein ,Fach' nicht dasselbe wie die für das gleiche Modell: So folgt das Fach Kulturwissenschaft in Tübingen anderen Traditionen als an der HU Berlin; so kann Kulturwissenschaft einmal mehr Europäische Ethnologie, einmal mehr Kulturgeschichte sein; oder sie ist ein Programmstudium verschiedener Disziplinen wie in Frankfurt/Oder. Ferner kann es auch sein, dass an derselben Universität Gender, Medien oder Kulturwissenschaft sowohl als Orientierungen bestehender Fächer wie auch als Einzelfächer eingerichtet werden. Kulturwissenschaft(en) und cultural studies weisen unterdessen derart ausdifferenzierte Theorien, Modelle und (!) Gegenstandfelder auf, dass verschiedene disziplinäre oder nicht-disziplinäre, also konkurrierende Lösungen das Beste sind. Dies stellt die offene experimentelle Situation her, die man wollen muss, um auf Dauer, durch vergleichende Evaluation, aus dem Set der Optionen ein oder mehrere Modelle favo- risieren zu können. Bei der relativen Neuheit von Medienwissenschaft, Gender Studies oder Kulturwissenschaft an bundesdeutschen Universitäten ist das Offenhalten dieses kognitiven und methodologischen Experimentalfeldes genau das Richtige. Übrigens gilt Ähnliches für die Wissenschaftsgeschichte, die performance studies, die Semiotik: Sie sind mal eine ,Orientierung', mal ein ,Fach', mal ein ,Feld' und dies jeweils anders in den USA oder in Deutschland.

\section{Literaturverzeichnis}

Allerkamp, Andrea/Raulet, Gérard (Hgg.) (2010): Kulturwissenschaften in Europa - eine grenzüberschreitende Disziplin? Münster: LIT.

Arendt, Hannah (1998): Vom Leben des Geistes. München: Piper.

Arendt, Hannah (1981): Vita activa oder Vom tätigen Leben [zuerst engl. 1958]. München: Piper.

Assmann, Aleida (2006): Einführung in die Kulturwissenschaft: Grundbegriffe, Themen, Fragestellungen. Berlin: E. Schmidt.

Bachmann-Medick, Doris (2006): Cultural turns. Neuorientierung in den Kulturwissenschaften. Reinbek bei Hamburg: Rowohlt

Beard, Georg Miller (1880): A Practical Treatise on Nervous Exhaustion (Neurasthenia), Its Symptoms, Nature, Sequences, Treatment. New York.

Beard, George Miller (1881): American Nervousness. Its Causes and Consequences. New York: Putnam.

Beck, Ulrich (1987): Risikogesellschaft. Auf dem Weg in eine andere Moderne. Frankfurt a.M.: Suhrkamp.

Beck, Ulrich (2007): Weltrisikogesellschaft. Auf der Suche nach der verlorenen Sicherheit. Frankfurt a.M.: Suhrkamp.

Benthien, Claudia/Velten, Hans Rudolf (Hgg.) (2002): Germanistik als Kulturwissenschaft. Reinbek bei Hamburg: Rowohlt.

Bernstein, Peter L. (1997): Wider die Götter. Die Geschichte von Risiko und Riskmanagement von der Antike bis heute. München: Gerling.

Böhme, Hartmut (2002): Religion und Moderne. In: Gräb, Wilhelm/Weyel, Birgit (Hgg.): Praktische Theologie und protestantische Kultur. Gütersloh: Kaiser, Gütersloher Verlagshaus, S. 17-35.

Böhme, Hartmut (2006): Religion und Kulturtheorie. In: Gräb, Wilhelm/Weyel, Birgit (Hg.): Religion in der modernen Lebenswelt. Erscheinungsformen und Reflexionsperspektiven. Gütersloh: Kaiser, Gütersloher Verlagshaus, S. 208-230.

Böhme, Hartmut (2008): Vom "turn" zum „vertigo". Wohin drehen sich die Kulturwissenschaften? Rezension zu: Doris Bachmann-Medick: „Cultural Turns. Neuorientierung in den Kulturwissenschaften". In: JLT online (19.05.2008) = Journal of Literary Theory (JLT) 2. 
Böhme, Hartmut/Matussek, Peter/Müller, Lothar (2007): Orientierung Kulturwissenschaft. Was sie kann, was sie will. 3. Aufl. Reinbek bei Hamburg: Rowohlt.

Bourdieu, Pierre (1983): Ökonomisches Kapital, kulturelles Kapital, soziales Kapital. In: Kreckel, Reinhard (Hg.): Soziale Ungleichheiten. Göttingen: Schwartz 1983, S. 183-198 [= Soziale Welt, Sonderband 2].

Bourdieu, Pierre (1987): Die feinen Unterschiede. Kritik der gesellschaftlichen Urteilskraft. Frankfurt a.M.: Suhrkamp.

Conermann, Stephan (Hg.) (2012): Was ist Kulturwissenschaft? Zehn Antworten aus den "kleinen Fächern". Bielefeld: transcript.

Constable, Liz/Denisoff, Dennis/Potolsky, Mathew (Hgg.) (1998): Perennial Decay: On the Aesthetics and Politics of Decadance. University of Pennsylvania Press.

Csáky, Moritz/Leitgeb, Christoph (Hgg.) (2009): Kommunikation, Gedächtnis, Raum. Kulturwissenschaften nach dem "spatial turn". Bielefeld: transcript.

Därmann, Iris/Jamme, Christoph (Hgg.) (2007): Kulturwissenschaften. Konzepte, Theorien, Autoren. München: Fink.

Debord, Guy (1967): La Société du spectacle. Paris: Buchet/Castell.

Deleuze, Gilles (1996): Erschöpft. In: Beckett, Samuel (Hg.): Quadrat, Geister-Trio, ... nur noch Gewölk ..., Nacht und Träume. Frankfurt a.M.: Suhrkamp, S. 49-99.

Dreitzel, Hans Peter (1968): Die gesellschaftlichen Leiden und das Leiden an der Gesellschaft. Vorstudien zu einer Pathologie des Rollenverhaltens. Stuttgart: Encke.

Drost, Wolfgang (Hg.) (1986): Fortschrittsglaube und Dekadenzbewußtsein im Europa des 19. Jahrhunderts. Literatur - Kunst - Kulturgeschichte. Heidelberg: Winter.

Eco, Umberto (1985): Apokalyptiker und Integrierte. Zur kritischen Kritik der Massenkultur. Frankfurt a.M.: S. Fischer.

Ehrenberg, Alain (2004): Das erschöpfte Selbst. Depression und Gesellschaft in der Gegenwart. Frankfurt a.M.: Suhrkamp.

Felsch, Philipp (2006): Laborlandschaften. Physiologische Alpenreisen im 19. Jahrhundert. Göttingen: Wallstein.

Finke, Peter (2008): Kulturökologie. In: Nünning, Ansgar/ Nünning, Vera (Hg.): Einführung in die Kulturwissenschaften. Stuttgart: Metzler, S. 248-279.

Frühsorge, Gotthardt (1993): Die Kunst des Landlebens. Vom Landschloß zum Campingplatz. Eine Kulturgeschichte. München, Berlin: Koehler \& Amelang.

Geisen, Thomas (2011): Arbeit in der Moderne. Ein dialogue imaginaire zwischen Karl Marx und Hannah Arendt. Wiesbaden: Springer.

Hampe, Michael (2006): Die Macht des Zufalls. Vom Umgang mit dem Risiko. Berlin: wjs.

Han, Byung-Chul (2009): Duft der Zeit. Ein philosophischer Essay zur Kunst des Verweilens. Bielefeld: transcript.
Han, Byung-Chul (2010): Müdigkeitsgesellschaft. Berlin: Matthes \& Seitz.

Handke, Peter (1989): Versuch über die Müdigkeit. Frankfurt a.M.: Suhrkamp.

Heidegger, Martin: Gelassenheit. In: Gesamtausgabe Bd. 16. Frankfurt a.M.: Klostermann 1994, S. 517-529.

Hobuß, Steffi/Tams, Nicola (Hgg.): Lassen und Tun. Kulturphilosophische Debatten zum Verhältnis von Gabe und kulturellen Praktiken. Bielefeld: transcript 2014.

Hofmannsthal, Hugo von (1979): Manche freilich. In: ders.: Gesammelte Werke in zehn Bdn. Hg. v. Bernd Schoeller. Gedichte Dramen I. 1891-1898. Frankfurt a.M.: S. Fischer.

Kittsteiner, Heinz Dieter (Hg.) (2004): Was sind Kulturwissenschaften? 13 Antworten. München: Fink.

Krämer, Sybille/Bredekamp, Horst (Hg.)(2003): Bild Schrift - Zahl. München: Fink.

Kusber, Jan u.a. (Hgg.) (2010): Historische Kulturwissenschaften. Positionen, Praktiken und Perspektiven. Bielefeld: transcript.

Lafargue, Paul (1966): Das Recht auf Faulheit \& Persönliche Erinnerungen an Karl Marx. Frankfurt a.M.: Europäische Verlagsanstalt.

Leggewie, Claus/Lang, Anne-Katrin/Zifonun, Darius (Hgg.) (2010): Schlüsselwerke der Kulturwissenschaften. Bielefeld: transcript.

Liebsch, Burkhard/Rüsen, Jörn/Straub, Jürgen (Hgg.) (2004): Handbuch der Kulturwissenschaften. 3 Bde. Stuttgart/Weimar: Metzler.

Luhmann, Niklas (1991): Soziologie des Risikos; Berlin New York: de Gruyter.

Mahoney, Kristin (2015): Literature and the Politics of Post-Victorian Decadence. New York: Cambridge University Press.

Mersch, Dieter: Was sich zeigt. Materialität, Präsenz, Ereignis. München: Fink 2002.

Mosso, Angelo (1892): Die Ermüdung. Leipzig: S. Hirtzel.

Münkler, Herfried/Bohlender, Matthias/Meurer, Sabine (Hgg.) (2010): Sicherheit und Risiko. Über den Umgang mit Gefahr im 21. Jahrhundert. Bielefeld: transcript.

Münkler, Herfried/Bohlender, Matthias/Meurer, Sabine (Hgg.) (2010a): Handeln unter Risiko. Gestaltungsansätze zwischen Wagnis und Vorsorge. Bielefeld: transcript.

Neswald, Elizabeth (2006): Thermodynamik als kultureller Kampfplatz. Zur Faszinationsgeschichte der Entropie 1850-1915. Freiburg: Rombach.

Nietzsche, Friedrich (1988): Sämtliche Werke. Kritische Studienausgabe (= KSA). Hg. von Giorgio Colli und Mazzino Montinari. München/Berlin New York: de Gruyter.

Nordau (2012): Entartung. 2 Bde. [zuerst: Berlin 1892/93]. Berlin/Boston: de Gruyter.

Nünning, Ansgar (Hg.) (2005): Grundbegriffe der Kulturtheorie und Kulturwissenschaft. Stuttgart: Metzler.

Pick, Daniel (1993): Faces of Degeneration: A European Disorder, c. 1848 - c. 1918 . Cambridge University Press. 
Pross, Caroline (2013): Dekadenz: Studien zu einer großen Erzählung der frühen Moderne. Göttingen: Wallstein.

Rabinbach, Anson (1992): The Human Motor. Energy, Fatigue, and the Origins of Modernity. Berkeley, Los Angeles: University of California Press [dt.: Motor Mensch. Kraft, Ermüdung und die Ursprünge der Moderne. Wien: Turia + Kant 2001].

Radkau, Joachim (1994): Die wilhelminische Ära als nervöses Zeitalter, oder: Die Nerven als Netz zwischen Tempo- und Körpergeschichte. In: Geschichte und Gesellschaft 20. S. 211-241.

Radkau, Joachim (1998): Das Zeitalter der Nervosität. Deutschland zwischen Bismarck und Hitler. Darmstadt: Wissenschaftliche Buchgesellschaft.

Roelcke, Volker (1999): Krankheit und Kulturkritik. Psychiatrische Gesellschaftsdeutungen im bürgerlichen Zeitalter (1790 bis 1914). Frankfurt a.M: Campus.

Rousseau, Jean-Jacques (2012): Träumereien eines einsam Schweifenden. Nach dem Ms. und den Spielkarten neu übers., komment. und mit einem Nachw. vers. von Stefan Zweifel. Berlin: Matthes \& Seitz.

Scheler, Max (1955): Mensch und Geschichte. In: Gesammelte Werke, Bd. IX: Späte Schriften [zuerst 1929]. Bonn: Bouvier.

Sengle, Friedrich (1963): Wunschbild Land und Schreckbild Stadt. In: Studium Generale 16, S. 619-631.

Semper, Gottfried (1860/63): Der Stil in den technischen und tektonischen Künsten oder praktische Ästhetik. Ein Handbuch für Techniker, Künstler und Kunstfreunde. 2 Bde. München: Bruckmann.
Semper, Gottfried (1852): Wissenschaft, Industrie und Kunst. Vorschläge zur Anregung nationalen Kunstgefühls, bei dem Schlusse der Londoner Industrie-Ausstellung, London, den 11. October 1851. Braunschweig: Vieweg.

Sherry, Vincent B. (2015): Modernism and the reinvention of decadence. New York: Cambridge University Press.

Sloterdijk, Peter (2001): Streß und Freiheit. Frankfurt a. M.: Suhrkamp.

Söllner, Alfons (1990): Hannah Arendts, Vita Activa oder Vom tätigen Leben'. Ein Lektüreversuch. In: König, Helmut/Schauer, Helmut/Greiff, Bodo von (Hgg.): Sozialphilosophie der industriellen Arbeit. Wiesbaden: Springer, S. 200-226.

Söllner, Alfons (1996): Deutsche Politikwissenschaftler in der Emigration: Studien zu ihrer Akkulturation und Wirkungsgeschichte. Mit einer Bibliographie. Opladen: Westdeutscher Verlag.

Voigt, Dieter/Meck, Sabine: Gelassenheit. Geschichte und Bedeutung. Darmstadt 2005.

Wirth, Uwe (Hg.) (2008): Kulturwissenschaft. Eine Auswahl grundlegender Texte. Frankfurt a.M.: Suhrkamp.

Zapf, Hubert (2008): Kulturökologie und Literatur. Ein transdisziplinäres Paradigma der Literaturwissenschaft. In: ders. (Hg.): Kulturökologie und Literatur. Beiträge zu einem transdisziplinären Paradigma der Literaturwissenschaft. Heidelberg: Winter, S. 15-44.

Zimmermann, Harm-Peer (Hg.) (2007): Empirische Kulturwissenschaft, Europäische Ethnologie, Kulturanthropologie, Volkskunde. Leitfaden für das Studium einer Kulturwissenschaft an deutschsprachigen Universitäten. Marburg: Jonas. 